\title{
Performance Evaluation of Modified DBLA Using Dark Channel Prior \& CLAHE
}

\author{
Kirandeep Kaur \\ GIMET college/E.C.E, Amritsar, 143501, India \\ E-mail: kirangill2011@gmail.com \\ Neetu Gupta \\ Asst. Prof., GIMET college/E.C.E, Amritsar, 143501, India \\ E-mail: gmetneetugupta@gmail.com
}

\begin{abstract}
This paper has focused on the different image enhancement techniques. Image enhancement has found to be one of the most important vision applications because it has ability to enhance the visibility of images. It enhances the quality of poor pictures. Distinctive procedures have been proposed so far for improving the quality of the digital images. To enhance picture quality image enhancement can specifically improve and limit some data presented in the input picture. It is a kind of vision system which reductions picture commotion, kill antiquities, and keep up the informative parts. Its object is to open up certain picture characteristics for investigation, conclusion and further use. The main objective of this paper is to modify the DBLA using the dark channel prior and CLAHE to enhance the results further. The comparative analysis has shown the significant improvement over the CLAHE and the DBLA.
\end{abstract}

Index Terms- Image Enhancement, Human Visual Perception, DBLA, CLAHE, RGB.

\section{INTRODUCTION}

Producing digital images with good brightness/ contrast and detail is a strong requirement in several areas like visualization, remote sensing, biomedical image examination, error recognition. The primary constraint for almost all vision and image processing task is produce natural images and transforming the image such as to enhance the visual information. Image enhancement is used to accentuate and sharpen image features for demonstrate and analysis. Image enhancement is the practice of applying these techniques to assist the improvement of a result to a computer imaging crisis. These methods are function exact and developed experimentally. These kinds of methods include point operation, where each pixel is customized according to a fastidious equation that is independent on other pixel values; mask operations, where each pixel is customized according to the values of the pixel's neighbors; or global operations, where all the pixels values in the image are taken into concern. Spatial domain processing methods take account of all three types, but frequency domain operations, by character of the frequency transforms, are universal operations. Frequency domain operations are able to develop into mask operations, based only on a local neighbourhood, by performing the transform on small image blocks rather than whole image. For easy visual task enhancement is used as a preprocessing step in some computer vision applications, for example, to enhance the edges of an object or it is also used in applications where human screening of an image is essential before additional processing. It is also applicable for post processing process to generate a visually desirable image. In short image enhancement is a method to make images look better visually. For the contrast enhancement method, CLAHE method is used that to avoid the problems of $\mathrm{HE}$ and AHE that is amplification of the noise. This paper represents the integrated approach to overcome the problems of existing techniques. In this perform a contrast enhancement by using the integrated approach using dominant brightness level analysis, contrast limited adaptive histogram equalization and dark channel prior algorithm.

\section{CONTRAST LIMITED ADAPTIVE HISTOGRAM EQUALIZATION}

Contrast limited adaptive histogram equalization prevent the amplification by using contrast limiting. The contrast limiting procedure has to be applied for each neighborhood from which a transformation function is derived in contrast limiting adaptive histogram equalization. CLAHE does not operates on entire image, it is applicable for small regions that known as tiles. Contrast of small region (tiles) is enhanced. After matching histogram of the output region is then combined by using bilinear interpolation to abolish artificially induced boundaries. The contrast of homogeneous areas can be limited because it can reduce the amplification of noise that may occur in the image. CLAHE was basically introduced for medical imaging and it was successful to enhance the low contrast images such as portal films.

In this method no prior weather information is required to advance the quality of the image. In this the captured image is converted from RGB (red, green, and blue) colour space to the HIS (hue, saturation and intensity). This conversion is done because HIS colour space senses the colour same as the human eye. The HIS color model 
defines every color with hue, saturation and intensity components. After the conversion from RGB to HIS a relative region is set and then CLAHE method applies histogram equalization to a background region. Each pixel in the original image is in the center of background region. The original histogram is clipped and the clipped pixels are redistributed to each gray level. The new histogram is different with ordinary histogram, because pixel intensity is limited to a user-selectable maximum. First of all the average number of pixels are calculated according to the equation:

$$
N_{\text {avg }}=\frac{N_{C R-X P} \times N_{C R-Y P}}{N_{\text {gray }}}
$$

Where $N_{\text {avg }}$ an average number of pixels are, $N_{\text {gray }}$ is number of gray pixels in background region, $N_{C R-X P}$ is number of pixels in $\mathrm{x}$ dimension of background region, $N_{C R-Y P}$ and is number of pixels in $\mathrm{y}$ dimension of background region. The actual clip limit can be calculated as:

$$
N_{C L}=N_{\text {clip }} \times N_{\text {avg }}
$$

Where $N_{C L}$ is actual clip limit? The original histogram is clipped and the clipped pixels are redistributed to each gray- level.

\section{A. CLAHE on RGB color model}

RGB color space describes colors in terms of the amount of red $(R)$, green $(G)$ and blue $(B)$ present. It uses additive color mixing, because it depicts that which type of light require to be emitted to create a given color. Light is added to create form from out of the darkness.

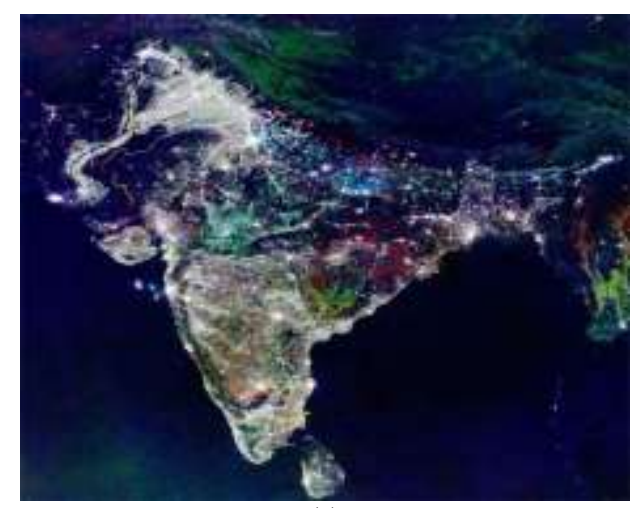

(a)

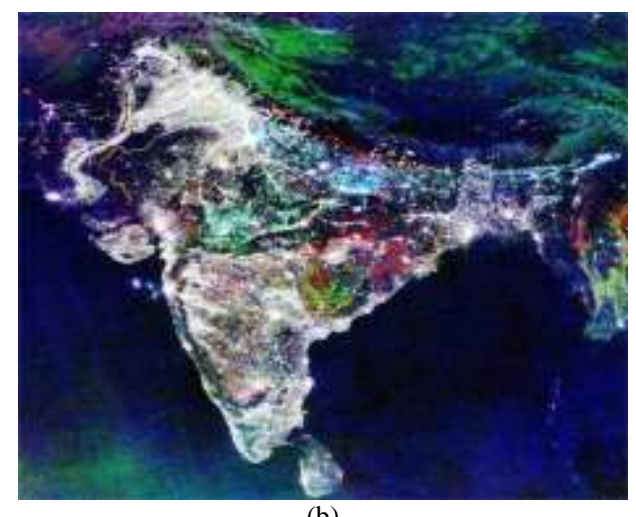

(b)

Fig. 1. shows the effect of CLAHE on RGB color model (a) Input image (b) CLAHE on RGB
The value of $R, G$, and $B$ components is the sum of the respective sensitivity functions and the incoming light.

$$
\begin{aligned}
& R=\int_{300}^{830} S(\gamma) R(\gamma) d \gamma \\
& G=\int_{300}^{830} S(\gamma) G(\gamma) d \gamma \\
& B=\int_{300}^{830} S(\gamma) B(\gamma) d \gamma
\end{aligned}
$$

where $\mathrm{S}(\gamma)$ is the light spectrum, $\mathrm{R}(\gamma), \mathrm{G}(\gamma), \mathrm{B}(\gamma)$ are the sensitivity functions for the $R, G$ and $B$ sensors respectively. In RGB color space, CLAHE can be applied on all the three components individually. The result of full-color RGB can be obtained by combining the individual components.

\section{DARK CHANNEL PRIOR}

Dark channel prior is a statistics of outdoor image haze removal. It is based on the fact that the local patches in the haze free images whose intensity is very low in at least one color channel. This information is used by dark channel prior method to improve the quality of image. From these pixels whose intensity is low the thickness of the haze can be estimated and the high quality haze free image can be recovered. High quality depth map of the image can also be obtained in this method [2]. The dark channel prior is based on the observation on fog-free outdoor images that there is at least one color channel having very low intensity at some pixels. For an image J we define dark channel as:

$$
J^{\text {dark }}(x)=\min _{c \in\{r g b\}}\left(\min _{y \in \Omega(x)}\left(j^{c}(y)\right)\right.
$$

Where $J^{c}$ a color channel of $\mathrm{J}$ and $\Omega(\mathrm{x})$ is a local patch centered at $x$. If $J$ is a fog-free outdoor image then excluding the sky area, the intensity of $J^{\text {dark }}$ is low that mean approximately zero. We call $J^{\text {dark }}$ the dark channel of J. Due to the air light; a foggy image is brighter than its fog-free version in where the transmission $t$ is low.

So the dark channel of the foggy image will have higher intensity in regions with denser fog. Thickness of the fog is a rough estimate of the intensity of the dark channel.

\section{DOMINANT BRIGHTNESS LEVEL ANALYSIS}

As increasing demand for enhancing remote sensing images, presented histogram-based contrast enhancement methods notable to conserve edge details and demonstrate saturation artifacts in low and high intensity regions. If do not consider spatially varying intensity distributions, in the same way contrast-enhanced images may have intensity distortion and lose image details in some regions[1].

For overcoming these problems, decompose the input image into four different layers of single dominant brightness levels. Perform the DWT on the input remote, 
sensing image and then estimate the dominant brightness level using the log average luminance in the LL sub band for this low frequency components used. The lowintensity layer has the dominant brightness lower than the pre specified low bound.

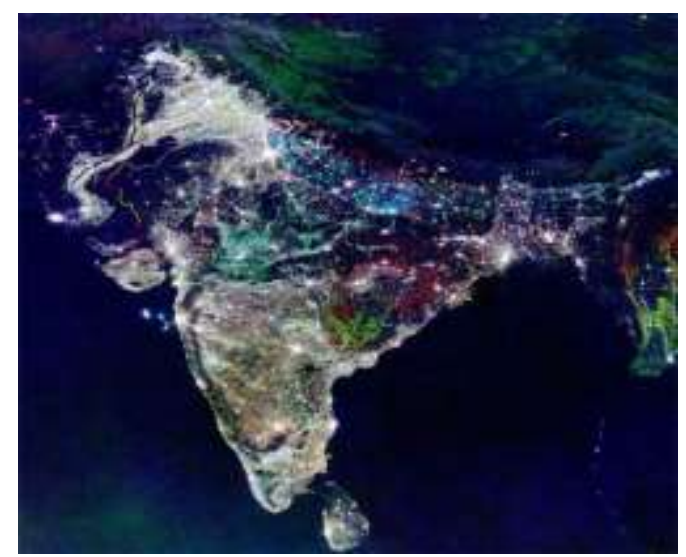

(a)

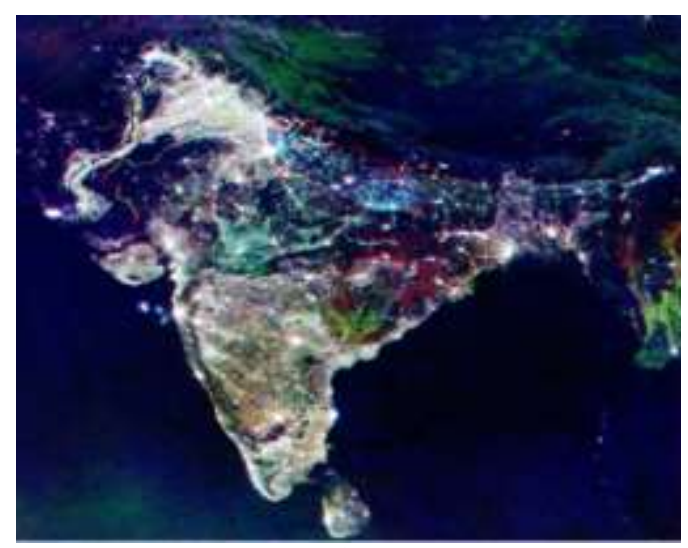

(b)

Fig. 2. shows the results of dominant brightness level analysis (a) Input image (b) Dominant brightness level analysis image

The high intensity layer is resolute in the similar manner with the pre specified high bound, and the middle-intensity layer has the dominant brightness in between low and high bounds [1].

Since high-intensity values are dominant in the bright region, and similarly for low -intensity values, the dominant brightness at the position $(x, y)$ is computed as:

$$
D(x, y)=\exp \left(\frac{1}{N L} \sum_{(x, y) \in S}\{\log L(x, y)+\varepsilon)\right.
$$

Where $S$ represents a rectangular region encompassing $(x, y), L(x, y)$ represents the pixel intensity at $(x, y), N L$ represents the total number of pixels in $S$, and $\varepsilon$ represents a satisfactorily small constant that prevents the log function from diverging to negative infinity. The lowintensity layer, high intensity layer and middle intensity layer has the dominant brightness level determined in the similar manner with pre specified low, high and middle bounds respectively. The normalized dominant brightness varies from zero to one, and it is practical range lies between 0.5 and 0.6 in most different images. For safely including the practical range of dominant brightness, we prefer 0.4 and 0.7 for the low and high bounds.

\section{GAPS IN SURVEY}

Following are the major disadvantages that are found in the related work of image enhancement techniques.

1. The survey has found that the most existing techniques are based upon the transform domain methods, which may introduce the color artifacts.

2. Transform domain method may reduce the intensity of the input remote sensing image.

3. The use of dark channel and CLAHE in algorithm ignored by many researchers to reduce the problem of poor brightness which will be presented in the output image due to dominant level.

\section{PROBLEM DEFINITION}

Image enhancement is one of the most popular algorithms used in vision applications for improving the visibility of the digital images. Recently much work is done in the field of remote sensing images to improve the visibility for improving their accuracy for further applications. Many algorithms have been proposed so far for enhancing the remote sensing images. It has been found that the most of the existing researchers have neglected many issues; i.e. no technique is accurate for different kind of circumstances. The existing methods have neglected the use of Contrast limited adaptive histogram equalization (CLAHE) to reduce the problem of poor brightness which will be presented in the image due to poor weather conditions. It is also found that the color artifacts which will be presented in the output image due to the transform domain methods; also neglected by the most of the researchers. So the present research work will use dark channel prior as the post processing function to enhance the results further.

\section{RESEARCH METHODOLOGY AND EXPERIMENTAL SET}

UP

In order to implement the proposed algorithm, design and implementation has been done in MATLAB using image processing toolbox. In order to do cross validation we have also implemented the enhanced DCT based image fusion using nonlinear enhancement. The developed approach is compared against some wellknown image fusion techniques available in literature. After these comparisons, we are comparing proposed approach against DCT using some performance metrics. Result shows that our proposed approach gives better results than the existing techniques.

To attain the objective, step-by-step methodology is used in this dissertation. Sub sequent are different steps which are used to accomplish this work. Following are the various steps used to accomplish the objectives of the dissertation.

\section{Proposed algorithm}

Step 1: Input images: select an input image. This image may be satellite image, foggy image, roadside image, natural image. 
Step 2: Discrete wavelet transform: Now perform the DWT is a wavelet transform for which the wavelets are discretely sampled. Discrete wavelet transform captures both frequency and location information. Wavelet used for feature extraction, denoising, compression, face recognition, image super resolution.

Step3: Dominant brightness level analysis: This analysis is done to remove the problem of intensity distortion and lose image details due to contrast enhancement and divide image based on dominant brightness level analysis is into three intensity (low, middle, high).

Step4: adaptive intensity transfer function: then apply adaptive intensity transfer function on each layer and do boundary smoothing after smoothing weighting map estimation method applied on it.

Step5: contrast limited adaptive histogram equalization: then this technique is apply to prevent the limiting amplification and this also applicable for limiting the amplification of noise.

Step 6: inverse discrete wavelet transform: this technique is applied to reform the data.

Step 7: Dark channel prior: Basically this technique used for haze removal and improve the quality of image.

Step 8: final: Results has been shown in the form of contrast enhanced image.

\section{EXPERIMENTAL RESULTS}

Fig 3 has shown the input images for experimental analysis. The overall objective is to combine relevant information from multiple images that is more informative and suitable for both visual perception and further computer processing.

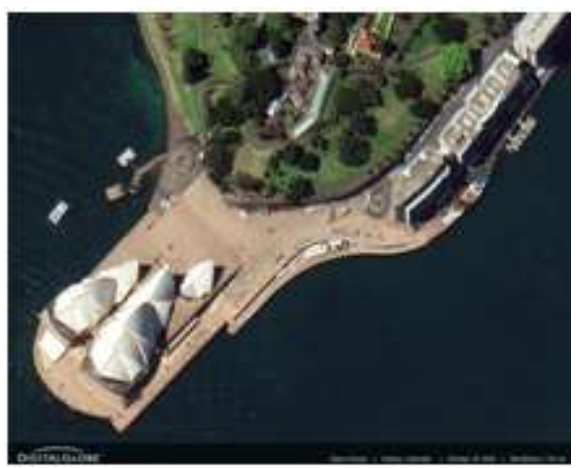

(a)

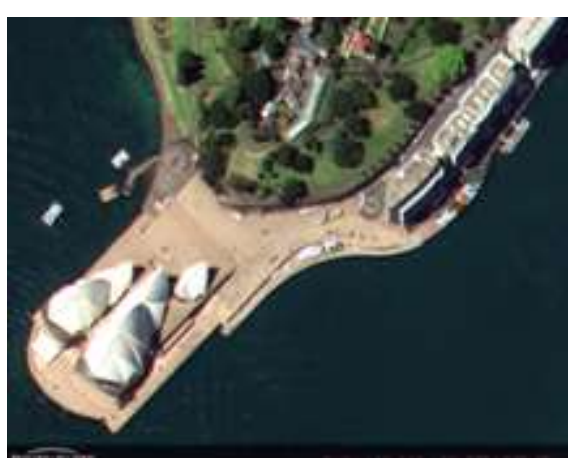

(b)

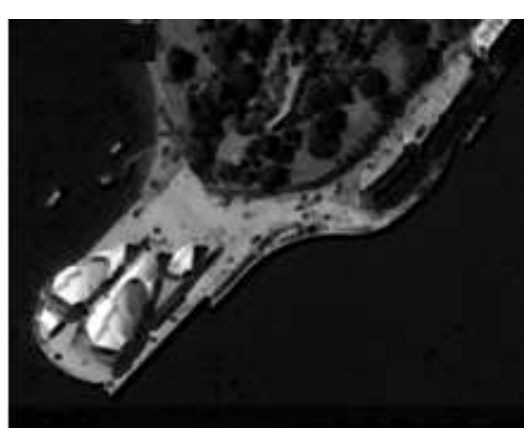

(c)

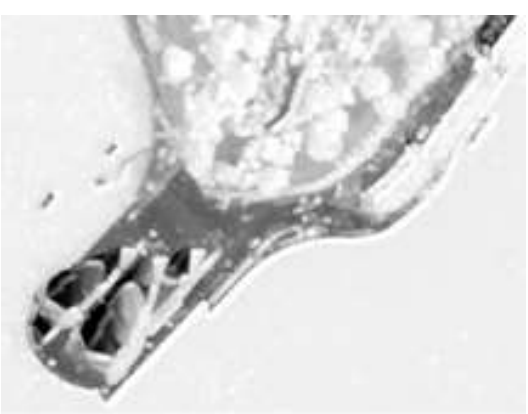

(d)

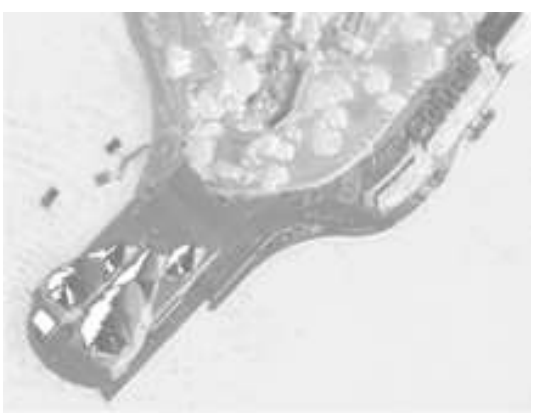

(e)

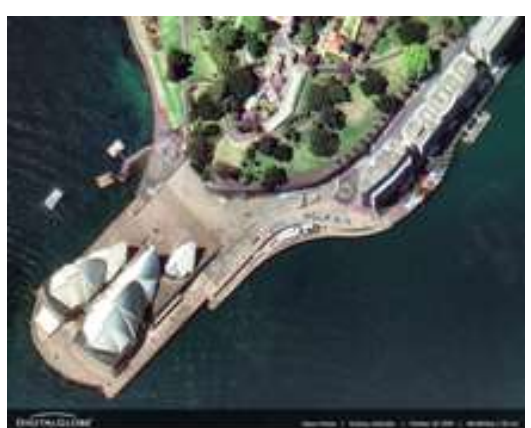

(f)

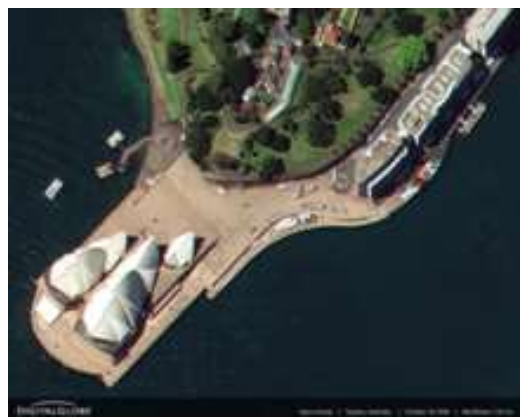

(g)

Fig. 3. represents the final results proposed methodology (a) Input image (b) Dominant brightness level analysis image (c) Depth map (d) Transmission map (e) (f) CLAHE on RGB color model image (g) final image 


\section{PERFORMANCE ANALYSIS}

This section shows the results between existing and proposed techniques. Some well-known image performance parameters for digital images have been selected to prove that the performance of the proposed algorithm is quite better than the existing methods.

\section{A. Mean square error}

One understandable mode of measuring this correspondence is to calculate an error signal by subtracting the test signal from the reference, and then computing the average energy of the error signal. The mean-squared-error (MSE) is the simplest, and the most widely used, full-reference image quality measurement. This metric is frequently used in signal processing and is defined as follows:

$$
M S E=\frac{1}{M N} \sum_{i=1}^{M} \sum_{j=1}^{N}(x(i, j)-y(i, j))^{2}
$$

Where $x(i . j)$ represents the original (reference) image and $y(i, j)$ represents the distorted (modified) image and $\mathrm{i}$ and $\mathrm{j}$ are the pixel position of the $\mathrm{M} \times \mathrm{N}$ image. MSE is zero when $x(i, j)=(i, j)$.

Table 1. Mean square error

\begin{tabular}{|c|c|c|c|}
\hline Image & DBL & RGB & PROPOSED \\
\hline 1. & 338 & 667 & 213 \\
\hline 2. & 428 & 635 & 339 \\
\hline 3. & 296 & 948 & 189 \\
\hline 4. & 388 & 267 & 54 \\
\hline 5. & 119 & 229 & 29 \\
\hline 6. & 372 & 105 & 38 \\
\hline 7. & 355 & 442 & 71 \\
\hline 8. & 478 & 968 & 404 \\
\hline 9. & 168 & 510 & 84 \\
\hline 10. & 607 & 989 & 88 \\
\hline 11. & 268 & 636 & 165 \\
\hline 12. & 356 & 1017 & 239 \\
\hline 13. & 237 & 559 & 56 \\
\hline 14. & 184 & 184 & 87 \\
\hline 15. & 549 & 692 & 470 \\
\hline
\end{tabular}

Comparative analysis of mean square error in Figure represent as:

Figure 1 has explained that quantized analysis of the mean square error. As mean square error need to be reduced therefore the proposed algorithm is showing the better results than the available methods as mean square error is less in every case.

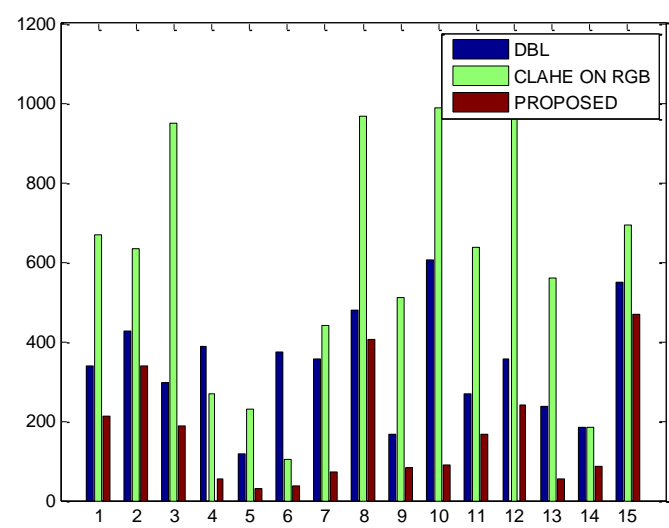

Fig. 1. Analysis of mean square error

\section{B. Peak signal to noise ratio}

Larger SNR and PSNR indicate a smaller difference between the original (without noise) and reconstructed image. This is the most widely used objective image quality/ distortion measure. The main advantage of this measure is ease of computation but it does not reflect perceptual quality. An important property of PSNR is that a slight spatial shift of an image can cause a large numerical distortion but no visual distortion and conversely a small average distortion can result in a damaging visual artifact, if all the error is concentrated in a small important region. This metric neglects global and composite error PSNR is calculated using Equation:

$$
P S N R=20 \log _{10}\left(\frac{N}{R M S E}\right) d b
$$

\begin{tabular}{|c|c|c|c|}
\hline Image & DBL & RGB & PROPOSED \\
\hline 1. & 22.8416 & 19.8895 & 24.8470 \\
\hline 2. & 21.8164 & 20.1031 & 22.1211 \\
\hline 3. & 23.4179 & 18.3627 & 25.3662 \\
\hline 4. & 22.2425 & 23.8657 & 30.8069 \\
\hline 5. & 27.3753 & 24.5324 & 33.5068 \\
\hline 6. & 22.4254 & 27.9189 & 32.3330 \\
\hline 7. & 22.6285 & 21.6766 & 29.6182 \\
\hline 8. & 21.3365 & 18.2721 & 22.0670 \\
\hline 9. & 25.8777 & 21.0551 & 28.8880 \\
\hline 10. & 20.2989 & 18.1788 & 28.6860 \\
\hline 11. & 23.8495 & 20.0962 & 25.9560 \\
\hline 12. & 22.6163 & 18.0576 & 24.3468 \\
\hline 13. & 24.3833 & 20.6567 & 30.6489 \\
\hline 14. & 25.4826 & 25.4826 & 28.7356 \\
\hline 15. & 20.7351 & 19.7297 & 21.4098 \\
\hline
\end{tabular}

Table 2. Peak to signal ratio

Comparative analysis of peak to signal ration in Figure represent as: 


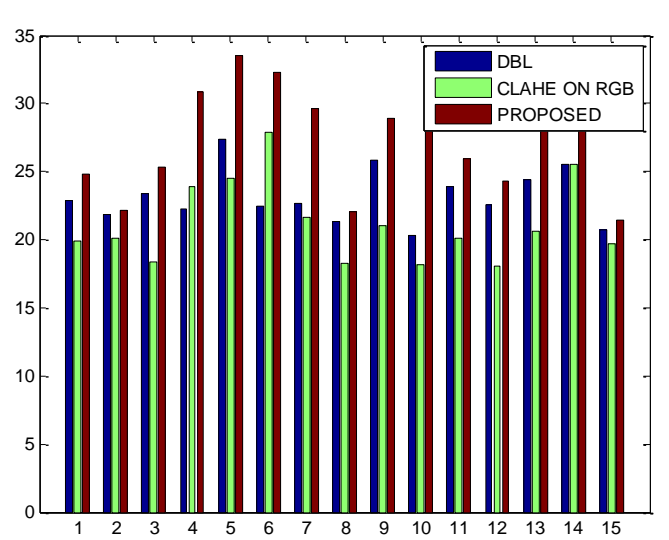

Fig. 2. Analysis of peak to signal ratio

Figure 2 is showing the comparative analysis of the Peak Signal to Noise Ratio (PSNR). As PSNR need to be maximized; so the main goal is to increase the PSNR as much as possible. Figure 2 has clearly shown that the PSNR is maximum in the case of the DBL technique therefore it is providing better results than the available methods.

\section{Root mean square error}

The root mean square error is a normally used to calculate of the difference between values predicted by a model and values actually observed from the surroundings that is being modeled. These individual differences are also called residuals, and the RMSE serves to total them into a single measure of analytical power.

Table 3. Root mean square error

\begin{tabular}{|c|c|c|c|}
\hline Image & DBL & RGB & PROPOSED \\
\hline 1. & 18.3848 & 25.8263 & 14.5945 \\
\hline 2. & 20.6882 & 25.1992 & 19.9750 \\
\hline 3. & 17.2047 & 30.7896 & 13.7477 \\
\hline 4. & 19.6977 & 16.3401 & 7.3485 \\
\hline 5. & 10.9087 & 15.1327 & 5.5852 \\
\hline 6. & 19.2873 & 10.2470 & 6.1644 \\
\hline 7. & 18.8414 & 21.0238 & 8.4261 \\
\hline 8. & 21.8632 & 31.1127 & 20.0998 \\
\hline 9. & 12.9615 & 22.5832 & 9.1652 \\
\hline 10. & 24.6374 & 31.4484 & 9.3808 \\
\hline 11. & 16.3767 & 25.2190 & 12.8452 \\
\hline 12. & 18.8680 & 31.8904 & 15.4596 \\
\hline 13. & 15.3948 & 23.6432 & 7.4833 \\
\hline 14. & 13.5647 & 13.5647 & 9.3274 \\
\hline 15. & 23.4307 & 26.3059 & 21.6795 \\
\hline
\end{tabular}

The RMSE of a model total with respect to the estimated variable $X_{\text {model }}$ is defined as the square root of the mean squared error:

$$
R M S E=\sqrt{\frac{\sum_{i=1}^{n}\left(X_{o b s, i}-X_{\text {model }_{i}}\right)^{2}}{n}}
$$

Where $X_{o b s}$ is observed values and $X_{\text {model }}$ is modeled values at time/place $i$. As RMSE need to be minimized; so the main goal is to decrease the RMSE as much as possible.

Comparative analysis of root mean square error in Figure represent as below:

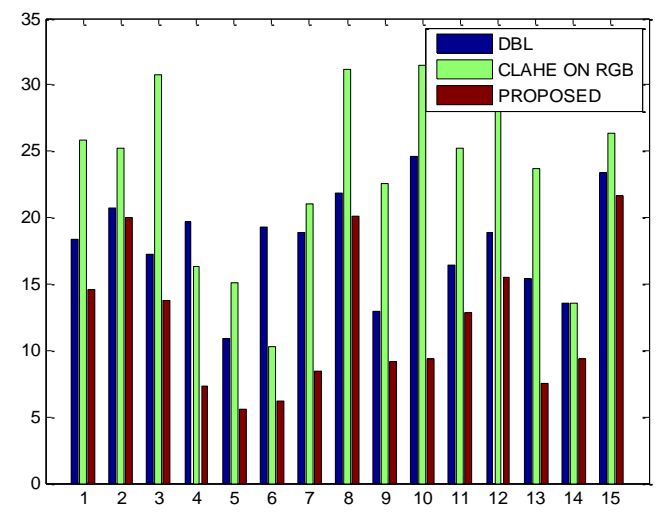

Fig. 3. Analysis of root mean square error

Figure 3 is showing the comparative analysis of the root mean square error (RMSE). As RMSE need to be minimized; so the main goal is to decrease the RMSE as much as possible. This is clearly shown that the RMSE is less in dominant brightness level.

\section{Bit error rate}

Bit error rate need to be minimized. Main goal is to decrease the BER as much as possible.

Table 4. Bit error rate

\begin{tabular}{|c|c|c|c|c|}
\hline Image & DBL & HSV & RGB & PROPOSED \\
\hline 1. & 0.0438 & 0.1426 & 0.0503 & 0.0402 \\
\hline 2. & 0.0458 & 0.0830 & 0.0497 & 0.0452 \\
\hline 3. & 0.0427 & 0.1316 & 0.0545 & 0.0394 \\
\hline 4. & 0.0450 & 0.3440 & 0.0419 & 0.0325 \\
\hline 5. & 0.0365 & 0.1175 & 0.0408 & 0.0298 \\
\hline 6. & 0.0446 & 0.3438 & 0.0358 & 0.0309 \\
\hline 7. & 0.0442 & 0.2779 & 0.0461 & 0.0338 \\
\hline 8. & 0.0469 & 0.1117 & 0.0547 & 0.0453 \\
\hline 9. & 0.0386 & 0.1137 & 0.0475 & 0.0346 \\
\hline 10. & 0.0493 & 0.964 & 0.0550 & 0.0349 \\
\hline 11. & 0.0419 & 0.1284 & 0.0498 & 0.0385 \\
\hline 12. & 0.0442 & 0.1382 & 0.0554 & 0.0411 \\
\hline 13. & 0.0410 & 0.1799 & 0.0484 & 0.0326 \\
\hline 14. & 0.0392 & 0.1201 & 0.0392 & 0.0348 \\
\hline 15. & 0.0482 & 0.1175 & 0.0507 & 0.0467 \\
\hline
\end{tabular}


Comparative analysis of Bit error rate in Figure represent as:

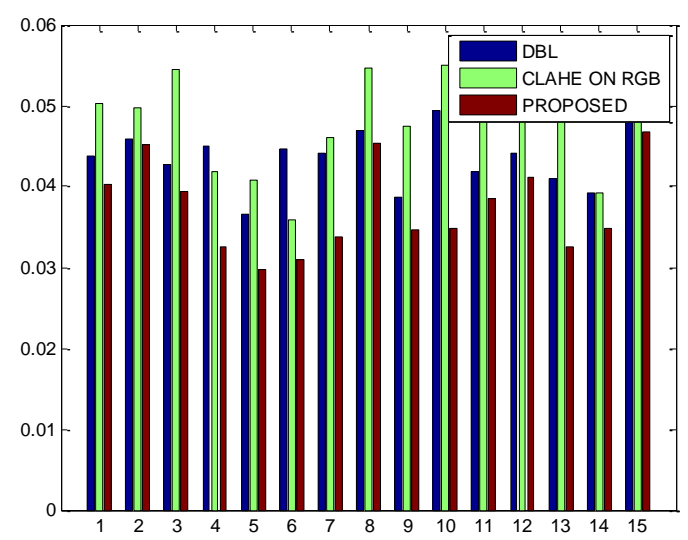

Fig. 4. Analysis of Bit error rate

Figure 4 is showing the comparative analysis of the BIT ERROR RATE (BER). As BER need to be minimized; so the main goal is to decrease the BER as much as possible. It has clearly shown that the BER minimum.

\section{E. Average Difference Evaluation}

Average difference is simply difference between the reference signal and test image. It is given by the equation.

$$
A D=\frac{1}{M N} \sum_{i=1}^{M} \sum_{j=1}^{N}(x(i, j)-y(i, j))
$$

As average difference needs to be minimized; so the main objective is to reduce the average difference as much as possible.

Table 5. Average Difference Evaluation

\begin{tabular}{|c|c|c|c|}
\hline Image & DBL & RGB & PROPOSED \\
\hline 1. & 10.3749 & 15.3312 & 0.0040 \\
\hline 2. & 2.6179 & 18.8196 & 0.0876 \\
\hline 3. & 8.4279 & 16.2675 & 0.0355 \\
\hline 4. & 17.5739 & 0.3007 & 0.0311 \\
\hline 5. & 7.0750 & 4.4720 & 0.0013 \\
\hline 6. & 17.6257 & 3.8261 & 0.0068 \\
\hline 7. & 16.1110 & 0.9463 & 0.0157 \\
\hline 8. & 6.9796 & 20.9881 & 0.0131 \\
\hline 9. & 7.9898 & 15.7940 & 0.0072 \\
\hline 10. & 22.3706 & 20.0926 & 0.0547 \\
\hline 11. & 9.5602 & 14.9370 & 0.0158 \\
\hline 12. & 10.0481 & 17.9571 & 0.0026 \\
\hline 13. & 11.9774 & 6.7968 & 0.0024 \\
\hline 14. & 8.7471 & 4.2851 & 0.0054 \\
\hline 15. & 4.7304 & 15.3147 & 0.0109 \\
\hline
\end{tabular}

Comparative analysis of root mean square error in Figure represent as below:

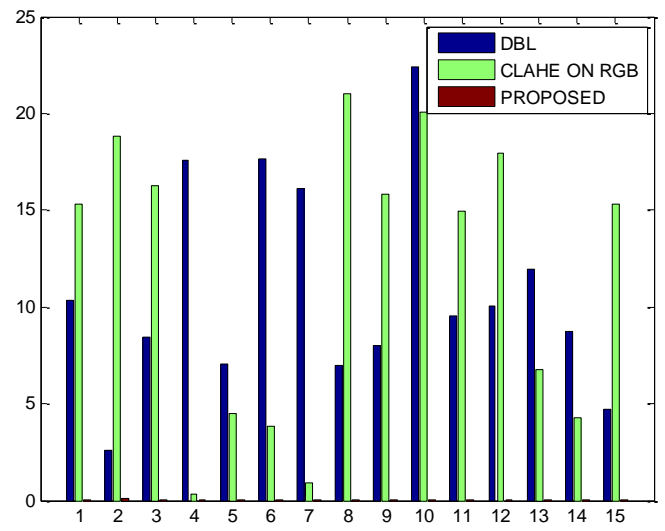

Fig. 5. Analysis of Average difference evaluation

Figure 5 is showing the comparative analysis of the AVERAGE DIFFERENCE EVALUTION (AD). As AD needs to be minimized; so the main goal is to decrease the $\mathrm{AD}$ as much as possible. It has clearly shown that the AD minimum.

\section{F. Structural content}

$\mathrm{SC}$ is also correlation based measure and measures the similarity between two images. Structural content is given by the equation

$$
S C=\frac{\sum_{i=1}^{M} \sum_{j=1}^{N}(y(i, j))^{2}}{\sum_{i=1}^{M} \sum_{j=1}^{N}(x(i, j))^{2}}
$$

As SC needs to be close to 1, therefore proposed algorithm is showing better results than the available methods as SC is close to 1 in every case.

Table 6. Structural content

\begin{tabular}{|c|c|c|c|}
\hline Image & DBL & RGB & PROPOSED \\
\hline 1. & 0.8367 & 0.7276 & 1.0102 \\
\hline 2. & 0.8866 & 0.5731 & 1.0559 \\
\hline 3. & 0.8357 & 0.7534 & 1.0099 \\
\hline 4. & 0.8273 & 1.0181 & 1.0014 \\
\hline 5. & 0.9603 & 0.9603 & 1.0016 \\
\hline 6. & 0.8298 & 1.0364 & 1.0007 \\
\hline 7. & 0.8270 & 1.0008 & 1.0017 \\
\hline 8. & 0.8278 & 1.2212 & 1.0285 \\
\hline 9. & 0.8338 & 0.7448 & 1.0081 \\
\hline 10. & 0.8276 & 1.1372 & 1.0018 \\
\hline 11. & 0.8354 & 0.7287 & 1.0095 \\
\hline 12. & 0.8374 & 0.6948 & 1.0109 \\
\hline 13. & 0.8283 & 1.0623 & 1.0018 \\
\hline 14. & 0.8321 & 0.9018 & 1.0059 \\
\hline 15. & 0.8558 & 0.7779 & 1.0243 \\
\hline & & & \\
\hline 1. & & 0.969 \\
\hline
\end{tabular}

Comparative analysis of structural content in Figure represent as below: 


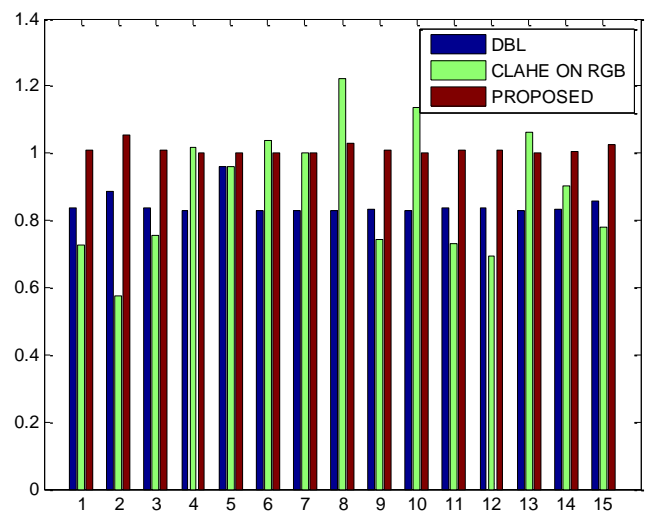

Fig. 6. Analysis of Structural content evaluation

Figure 6 is showing the comparative analysis of the STRUCTURAL CONTENT (SC). As SC needs to be one; so the main goal is to maintain the $\mathrm{SC}$ as much as possible to one. It has clearly shown that the SC one.

\section{G. Normalized cross correlation}

The closeness between two digital images can also be quantified in terms of correlation function. Normalized cross-correlation measures the similarity between two images and is given by the equation

$$
N K=\frac{\sum_{i=1}^{M} \sum_{j=1}^{N}(x(i, j) \times y(i, j))}{\sum_{i=1}^{M} \sum_{j=1}^{N}(x(i, j))^{2}}
$$

Table 7. Normalized cross correlation evaluation

\begin{tabular}{|c|c|c|c|}
\hline Image & DBL & RGB & PROPOSED \\
\hline 1. & 1.0846 & 1.1616 & 0.9867 \\
\hline 2. & 1.0117 & 1.2950 & 0.9248 \\
\hline 3. & 1.0853 & 1.1221 & 0.9868 \\
\hline 4. & 1.0986 & 0.9871 & 0.9985 \\
\hline 5. & 1.0082 & 1.0082 & 0.9976 \\
\hline 6. & 1.0960 & 0.9809 & 0.9991 \\
\hline 7. & 1.0991 & 0.9919 & 0.9979 \\
\hline 8. & 1.0978 & 0.6416 & 0.9619 \\
\hline 9. & 1.0899 & 1.1417 & 0.9911 \\
\hline 10. & 1.0983 & 0.9301 & 0.9983 \\
\hline 11. & 1.0862 & 1.1570 & 0.9877 \\
\hline 12. & 1.0827 & 1.1786 & 0.9850 \\
\hline 13. & 1.0971 & 0.9553 & 0.9975 \\
\hline 14. & 1.0913 & 1.0448 & 0.9925 \\
\hline 15. & 1.0545 & 1.1053 & 0.9627 \\
\hline
\end{tabular}

As NCC needs to be close to 1 , therefore proposed algorithm is showing better results than the available methods as NCC is close to 1 in every case.

Comparative analysis of normalized cross-correlation in Figure represent as below:

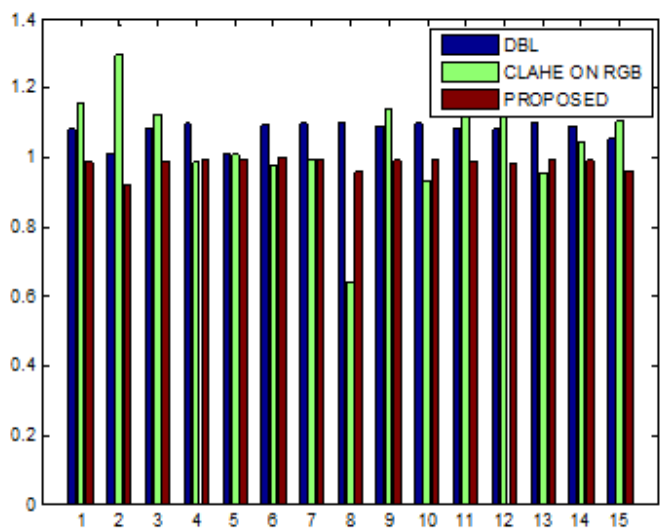

Fig. 7. Analysis of normalized cross-correlation evaluation

Figure 7 is showing the comparative analysis of the normalized cross-correlation (NCC). As NCC needs to be close to one; so the main goal is to maintain NCC as much as possible to close to one. It has clearly shown that the NCC is more close to one.

\section{CONCLUSION}

Dominant brightness level analysis has been used to overcome the problems of intensity distortion and lose image details in some regions of contrast enhanced images without considering the spatially varying intensity distributions. For overcoming these problems, decompose the input image into multiple layers of single dominant brightness levels. To use the low-frequency luminance components, perform the DWT on the input remote sensing image and then estimate the dominant brightness level using the log average luminance in the LL sub band. The low-intensity layer has the dominant brightness lower than the pre specified low bound. The high intensity layer is determined in the similar manner with the pre specified high bound, and the middleintensity layer has the dominant brightness in between low and high bounds. The survey has found that the most existing techniques are based upon the transform domain methods, which may introduce the color artifacts. Transform domain method may reduce the intensity of the input remote sensing image. The use of dark channel and CLAHE in algorithm is ignored by many researchers to reduce the problem of poor brightness which will be presented in the output image due to dominant level. The proposed technique has modified the DBLA using CLAHE and dark channel prior. The modified DBLA has the potential to overcome the limitations of the earlier work. The proposed technique has been designed and implemented in MATLAB using image processing toolbox. The comparison among CLAHE, DBLA and proposed has shown the significant improvement of the proposed technique.

\section{REFERENCES}

[1] Eunsung lee, Sangjin Kim, Wonseok Kang, Doochun Seo, Joonki Paik "Contrast Enhancement Using Dominant 
Brightness Level Analysis and Adaptive Intensity Transformation for Remote Sensing Images.” pp.62-66, 2013.

[2] Ullah, E., R. Nawaz, and J. Iqbal. "Single image haze removal using improved dark channel prior." Modelling, Identification \& Control (ICMIC), 2013 Proceedings of International Conference on. IEEE, 2013.

[3] Setiawan, Agung W., et al. "Color Retinal Image Enhancement using CLAHE." 2013 International Conference on ICT for Smart Society (ICISS).

[4] Agaian, Sos, and Mehdi Roopaei. "New haze removal scheme and novel measure of enhancement." Cybernetics (CYBCONF), 2013 IEEE International Conference on. IEEE, 2013.

[5] Hitam, M. S., et al. "Mixture contrast limited adaptive histogram equalization for underwater image enhancement." Computer Applications Technology (ICCAT), 2013 International Conference on. IEEE, 2013.

[6] Im, Jaehyun, et al. "Dark channel prior-based spatially adaptive contrast enhancement for back lighting compensation." Acoustics, Speech and Signal Processing (ICASSP), 2013 IEEE International Conference on. IEEE, 2013.

[7] Kil, Tae Ho, Sang Hwa Lee, and Nam Ik Cho. "A dehazing algorithm using dark channel prior and contrast enhancement." Acoustics, Speech and Signal Processing (ICASSP), 2013 IEEE International Conference on. IEEE, 2013.

[8] Zhu, Qingsong, et al. "An adaptive and effective single image dehazing algorithm based on dark channel prior." Robotics and Biomimetics (ROBIO), 2013 IEEE International Conference on. IEEE, 2013.

[9] Saleem, Amina, Azeddine Beghdadi, and Boualem Boashash. "Image fusion-based contrast enhancement." EURASIP Journal on Image and Video Processing 2012: pp.1-17, 2012.

[10] Khan, Mohd Farhan, Ekram Khan, and Z. A. Abbasi. "Weighted average multi segment histogram equalization for brightness preserving contrast enhancement." Signal Processing, Computing and Control (ISPCC), 2012 IEEE International Conference on. IEEE, 2012.

[11] Peng,Li,Chuangbai Xiao, and Jing Yu. "single image fog removal based on dark channel prior and localextrema." american journal of engineering and Technology Research Vol 12.2 (2012).

[12] Tripathi, A. K., and S. Mukhopadhyay. "Single image fog removal using anisotropic diffusion." Image Processing, IET 6.7 (2012): 966-975.

[13] Rajesh Garg, Bhawna Mittal, Sheetal Garg "Histogram Equalization Techniques For Image Enhancement" IJECT vol2, issue1, March 2011.

[14] Demirel, Hasan, Cagri Ozcinar, and Gholamreza Anbarjafari. "Satellite image contrast enhancement using discrete wavelet transform and singular value decomposition." Geoscience and Remote Sensing Letters, IEEE 7.2: pp.333-337, 2010.

[15] Guo, Fan, et al. "Automatic Image Haze Removal Based on Luminance Component." Wireless Communications Networking and Mobile Computing (WiCOM), 2010 6th International Conference on. IEEE, 2010.

[16] Yisu Zhao, Nicolas D. Georgans, Emil M. Petriu "Applying Contrast-Limited Adaptive Histogram Equalization and Integral Projection For Facial Feature Enhancement and Detection" 2010, IEEE.

[17] Chen, Mengyang, et al. "Single image defogging." Network Infrastructure and Digital Content,
2009. IC-NIDC 2009. IEEE International Conference on. IEEE, 2009.

\section{Authors' Profiles}

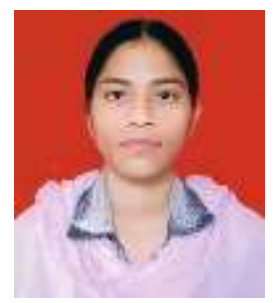

Kirandeep Kaur d/o Gurmukh Singh, D.O.B 29-10-1990, B.tech in ECE from SSIET, Patti, District TarnTaran Punjab, in 2011 with $71 \%$ marks and M.etch with $64 \%$ upto $3^{\text {rd }}$ semester from Global Institute of management and emerging Technologies Amritsar.

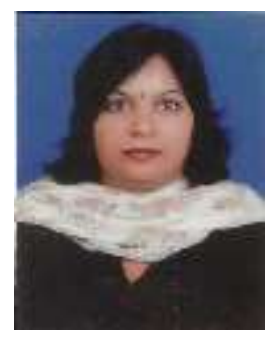

Neetu Gupta d/o Banwari Lal Gupta. D.O.B 15-5-1976, M.tech in ECE from Beant Collage of engeering and technology Gurdaspur in 2012 with $71 \%$ marks and has 8 year experience in teaching-4 year in Global institute of management and emerging Technologies and 4 year in Aman Bhalla Institutes.

How to cite this paper: Kirandeep Kaur, Neetu Gupta,"Performance Evaluation of Modified DBLA Using Dark Channel Prior \& CLAHE", International Journal of Intelligent Systems and Applications (IJISA), vol.7, no.5, pp.48-56, 2015. DOI: $10.5815 /$ ijisa.2015.05.07 